\title{
Long-lived entanglement generation of nuclear spins using coherent light
}

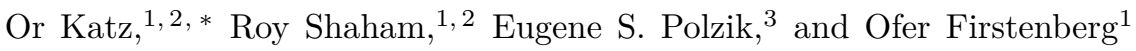 \\ ${ }^{1}$ Department of Physics of Complex Systems, Weizmann Institute of Science, Rehovot 76100, Israel \\ ${ }^{2}$ Rafael Ltd, IL-31021 Haifa, Israel \\ ${ }^{3}$ Niels Bohr Institute, University of Copenhagen, \\ Blegdamsvej 17, DK-2100 Copenhagen, Denmark.
}

\begin{abstract}
Nuclear spins of noble-gas atoms are exceptionally isolated from the environment and can maintain their quantum properties for hours at room temperature. Here we develop a mechanism for entangling two such distant macroscopic ensembles by using coherent (i.e. classical) light input. The interaction between the light and the noble-gas spins in each ensemble is mediated by spinexchange collisions with alkali-metal spins, which are only virtually excited. The relevant conditions for experimental realizations with ${ }^{3} \mathrm{He}$ or ${ }^{129} \mathrm{Xe}$ are outlined.
\end{abstract}

Quantum entanglement describes correlations between distinct quantum systems and is often used to set borders between the quantum and classical worlds [1, 2]. It is a valuable resource for quantum information and computing [3-7] and for metrology beyond the standard quantum limits [8, 9]. Generating and maintaining entanglement in matter systems requires exquisite control and isolation, as achieved in ensembles of alkali-metal spins [10-12, trapped ions and atoms [13, 14, quantum defects in crystals [15, and high-quality mechanical oscillators [16.

Rare isotopes of noble-gas atoms, such as ${ }^{3} \mathrm{He}$ and ${ }^{129} \mathrm{Xe}$, have nuclei with nonzero spins. These spins are exceptionally isolated from the environment and can remain coherent for extremely long times, exceeding tens of hours above room-temperature [17, 18. Accordingly, the collective nuclear spin of noble-gas ensembles is the longest-living macroscopic quantum object currently known. Nevertheless, while these spin ensembles could potentially maintain entanglement for record times [19, 20, they do not interact with optical photons. This limits their applicability for optical quantum communication [10, 21-24], or to advanced sensing applications such as hybrid optomechanical-spin systems, e.g., for gravitational-wave detection [25, 26]. In 2007, Pinard and coworkers proposed to entangle ${ }^{3} \mathrm{He}$ ensembles using incoherent collisions with metastable ${ }^{3} \mathrm{He}$ atoms and via adiabatic state transfer with nonclassical light in an optical cavity [27]. This pioneering and rather challenging proposal was never realized.

Here we develop a readily feasible scheme for entangling two macroscopic ensembles of noble-gas spins contained in distant cells, as shown in Fig. 1. Our scheme employs the archetypal mechanism for entanglement of spin ensembles, based on continuous measurement of spin fluctuations by off-resonant Faraday rotation of probe light [24. This mechanism was successfully employed to entangle distant alkali spin ensembles [10]. While there is no direct interaction between light and noble-gas spins, we propose to use auxiliary ensembles of alkali-metal atoms as mediators. The alkali mediators are opticallyaccessible and couple to the noble-gas spins via coherent

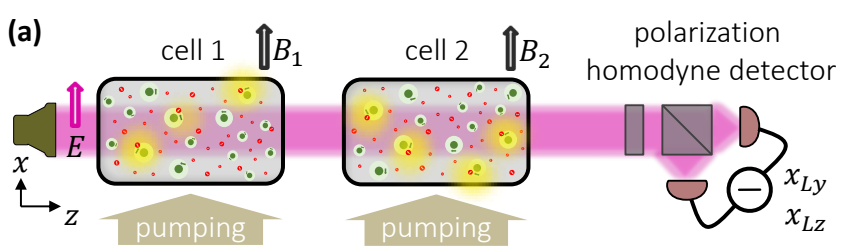

(b)

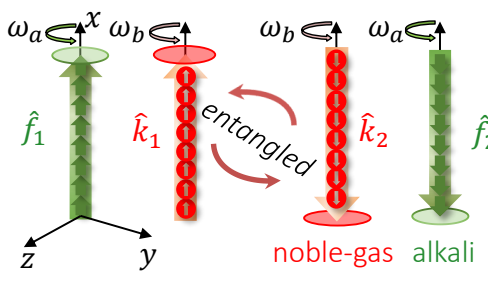

(c)

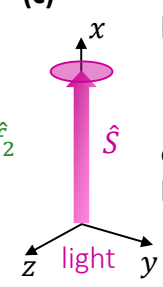

input $\hat{x}_{L Z}$ light

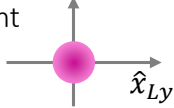

output $\hat{x}_{L Z}$ light

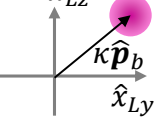

Figure 1. Entanglement generation of the macroscopic spinstate of two distant noble-gas ensembles. (a) The physical system consists of two cells with mixtures of alkali (green) and noble-gas atoms (red). Homodyne detection of coherent probe light passing through the two cells monitors the correlated spin precession of the noble-gas ensembles. (b) Collective spin-states of polarized alkali and noble-gas atoms. The shaded disks denote quantum spin fluctuations. (c) Polarization state of linearly-polarized probe, and its rotation via indirect Faraday interaction with the noble-gas spins, as described by Eq. 6. The in-phase $\left(\hat{x}_{L y}\right)$ and out-of-phase $\left(\hat{x}_{L z}\right)$ components of the probe commute and can be simultaneously measured. Shaded purple disks denote the photon shot-noise.

spin-exchange collisions [19]. We show that continuous optical measurement of the alkali spins generates a vital entanglement between the noble-gas ensembles. At the same time, dissipation and fluctuations of the alkali spins can be circumvented by introducing a frequency mismatch, such that quantum correlations are mediated without actual excitations of the (alkali) mediators. We outline the physical conditions for experiments with ${ }^{3} \mathrm{He}-$ $\mathrm{K}$ and ${ }^{129} \mathrm{Xe}-\mathrm{Rb}$ mixtures towards a demonstration of long-lived entanglement of macroscopic systems.

Before diving into the detailed model, we consider a simplified picture of the interaction mechanisms within each cell, presenting the emergence of the Faraday in- 


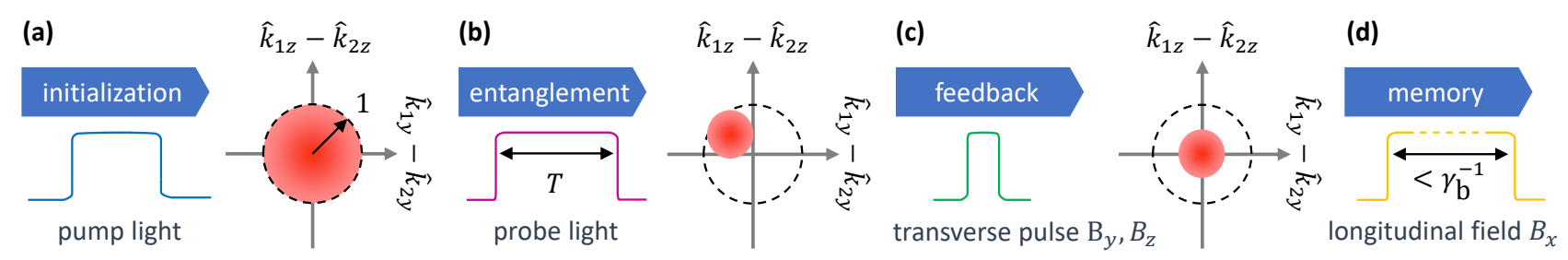

Figure 2. Sequence for generation and storage of entanglement. (a) The noble-gas ensembles are pumped to coherent spin states with vacuum fluctuations of radius $\operatorname{std}\left(\hat{\mathrm{k}}_{1 y}-\hat{\mathrm{k}}_{2 y}\right)=\operatorname{std}\left(\hat{\mathrm{k}}_{1 z}-\hat{\mathrm{k}}_{2 z}\right)=1$. Dashed circles mark the entanglement criterion from Eq. (1). (b) Homodyne detection of the probe light, via the Faraday interaction (Fig. 1. F), leads to (conditional) squeezing and displacement of the spin-state. $\hat{\mathrm{k}}_{1 y}-\hat{\mathrm{k}}_{2 y}$ and $\hat{\mathrm{k}}_{1 z}-\hat{\mathrm{k}}_{2 z}$ commute, and their combined uncertainty can be smaller than 1 . (c) A short transverse magnetic-field pulse rotates the spin-state, yielding an unconditioned entanglement, satisfying inequality (1). (d) During the memory time, application of a large magnetic-field decouples the noble-gas and alkali spins. The memory lifetime is governed by the long coherence-time of the noble-gas spins.

teraction between light and optically-inaccessible spins. We describe quantum excitations of the alkali spins by the bosonic operators $\hat{f}, \hat{f}^{\dagger}$, excitations of noble-gas spins by $\hat{k}, \hat{k}^{\dagger}$, and the polarization state of probe light by the canonical bosonic operators $\hat{x}_{\mathrm{L}}$ and $\hat{p}_{\mathrm{L}}$. The probe couples to the alkali ground-level spins via the opticallyexcited levels. These levels are subject to rapid relaxation at a rate $\Gamma_{e}$ due to spontaneous emission and buffer-gas broadening, leading to spin relaxation and to probe attenuation. Detuning the probe by $\left|\delta_{\mathrm{e}}\right| \gg \Gamma_{\mathrm{e}}$ from the optical transition circumvents this relaxation, rendering the atom-photon interaction dispersive. The excited-level spins then adiabatically follow the groundlevel spins, yielding the Faraday interaction $\mathcal{H}_{\mathrm{L}-\mathrm{a}}=$ $i \hbar Q \hat{p}_{\mathrm{L}}\left(\hat{f}^{\dagger}-\hat{f}\right) / \sqrt{2}$ between the probe and the alkali spins. $\mathcal{H}_{\mathrm{L}-\mathrm{a}}$ describes the polarization rotation of far-detuned probe and the resulting alkali-spin rotation at the rate $Q \propto 1 / \delta_{\mathrm{e}}[11$.

The coherent coupling of the alkali spins to the noble-gas spins is described by the exchange Hamiltonian $\mathcal{H}_{\mathrm{a}-\mathrm{b}}=\hbar J\left(\hat{f}^{\dagger} \hat{k}+\hat{k}^{\dagger} \hat{f}\right)$, where $J$ is the collective exchange-rate due to atomic collisions [19. The resonance conditions for this coupling are governed by the non-interacting Hamiltonian $\mathcal{H}_{0}=\hbar \omega_{\mathrm{a}} \hat{f}^{\dagger} \hat{f}+\hbar \omega_{\mathrm{b}} \hat{k}^{\dagger} \hat{k}$, where the difference in precession frequencies $\Delta=\omega_{\mathrm{a}}-\omega_{\mathrm{b}}$ is tunable with an external magnetic field.

The alkali spins are prone to fast dephasing at a rate $\gamma_{\mathrm{a}}$ due to photon absorption, collisions with different atoms and with the cell walls. Here again, the detuning $(\Delta)$ determines to what extent this fast alkali relaxation affects the noble-gas spins. On resonance $\left(|\Delta| \lesssim \gamma_{\mathrm{a}}, J\right)$, the noble-gas spins inherit the alkali-spin relaxation [19, whereas off resonance $\left(|\Delta| \gg J, \gamma_{\mathrm{a}}\right)$, the interaction is dispersive, suppressing the relaxation induced by the alkali by a factor $\gamma_{\mathrm{a}} / \Delta \ll 1$. The alkali spins then adiabatically follow the noble-gas spins, yielding the overall Hamiltonian $\mathcal{H}_{\mathrm{L}-\mathrm{b}}=i \hbar Q J \hat{p}_{\mathrm{L}}\left(\hat{k}-\hat{k}^{\dagger}\right) /(\sqrt{2} \Delta)$ in a frame rotating at $\omega_{\mathrm{b}}$ when $|\Delta| \gg J, Q$, up to shifts proportional to $Q^{2} / \Delta$ and $J^{2} / \Delta$. We thus arrive at an indirect Faraday interaction of light with noble-gas spins via virtual excitations of alkali spins.

The concept described above can be applied for entangling two distant noble-gas spin ensembles using probe light and alkali spins [Fig. 1(a)]. Each cell contains $N_{\mathrm{b}}$ noble-gas atoms with spin-1/2, initially polarized along the quantization axis $\boldsymbol{e}_{x}$. Ensemble $i=1,(i=2)$ is polarized upwards $+\boldsymbol{e}_{x}$ (downwards $-\boldsymbol{e}_{x}$ ). Given the spin operators $\hat{\mathbf{k}}_{i}^{(n)}$ of the $n$-th noble-gas atom in the $i$-th cell, we define the normalized macroscopic spin operator $\hat{\mathbf{k}}_{i} \equiv M_{\mathrm{b}}^{-1 / 2} \sum_{n=1}^{N_{\mathrm{b}}} \hat{\mathbf{k}}_{i}^{(n)}$ for each ensemble. The total magnetization $M_{\mathrm{b}}=P_{\mathrm{b}} N_{\mathrm{b}} / 2$ depends on the initial degree of polarization $P_{\mathrm{b}} \leq 1$. For $M_{\mathrm{b}} \gg 1$ and fully polarized ensembles $\left(P_{\mathrm{b}}=1\right)$, the initial states are known as coherent spin-states (CSS). A partially polarized ensemble of spin- $1 / 2$ atoms may be seen as a mixture of $P_{\mathrm{b}} N_{\mathrm{b}}$ polarized atoms and $\left(1-P_{\mathrm{b}}\right) N_{\mathrm{b}}$ unpolarized atoms, only reducing the coherent interaction strength [11. The two ensembles have definitive collective spin along $\boldsymbol{e}_{x}$ with a classical measurement outcome $\left\langle\hat{\mathrm{k}}_{i x}\right\rangle= \pm M_{\mathrm{b}}^{1 / 2}$ and negligible variance, where henceforth the symbol ' \pm ' stands for ' + ' in cell $i=1$, and for '- ' in cell $i=2$. On the other hand, the transverse components of the normalized collective spin $\hat{\mathrm{k}}_{i y}$ and $\hat{\mathrm{k}}_{i z}$ satisfy the commutation relation $\left[\hat{\mathrm{k}}_{i y}, \hat{\mathrm{k}}_{j z}\right]= \pm i \delta_{i j}$ and consequently are governed by quantum fluctuations. These operators are normalized and unitless, giving the collective spin variance in units of vacuum noise. These fluctuations, known as atom-projection noise, are zero on average and have a nonzero variance, satisfying the Robertson inequality $4 \operatorname{var}\left(\hat{\mathrm{k}}_{i y}\right) \operatorname{var}\left(\hat{\mathrm{k}}_{i z}\right) \geq\left|\left\langle\left[\hat{\mathrm{k}}_{i y}, \hat{\mathrm{k}}_{i z}\right]\right\rangle\right|^{2}=1$, where $\operatorname{var}\left(\hat{\mathrm{k}}_{i y}\right)=\operatorname{var}\left(\hat{\mathrm{k}}_{i z}\right)$ for CSS. Visually, these fluctuations can be represented as a small uncertainty disk around the classical spin vector, as shown in Fig. 1(b).

Two spin ensembles are entangled if their quantum fluctuations are correlated, as in a two-mode squeezed state. For spins of equal magnitude $\left|\left\langle\hat{\mathrm{k}}_{1 x}\right\rangle\right|=\left|\left\langle\hat{\mathrm{k}}_{2 x}\right\rangle\right|$, a sufficient criterion for EPR-type entanglement is given by [10, 28]

$$
\operatorname{var}\left(\hat{\mathrm{k}}_{1 y}-\hat{\mathrm{k}}_{2 y}\right)+\operatorname{var}\left(\hat{\mathrm{k}}_{1 z}-\hat{\mathrm{k}}_{2 z}\right)<2 .
$$


Therefore, simultaneous measurement of the nonlocal observables $\hat{\mathrm{k}}_{1 y}-\hat{\mathrm{k}}_{2 y}$ and $\hat{\mathrm{k}}_{1 z}-\hat{\mathrm{k}}_{2 z}$ generates entanglement, if the total noise variance of the two cells is less than two vacuum-noise units. Such measurement is allowed for oppositely oriented spins $\left\langle\hat{\mathrm{k}}_{1 x}\right\rangle=-\left\langle\hat{\mathrm{k}}_{2 x}\right\rangle$, for which $\hat{\mathrm{k}}_{1 y}-\hat{\mathrm{k}}_{2 y}$ and $\hat{\mathrm{k}}_{1 z}-\hat{\mathrm{k}}_{2 z}$ commute.

We measure the noble-gas spins using alkali spins and a probe field. Each cell contains $N_{\mathrm{a}}$ alkali atoms, polarized to a polarization degree $P_{\mathrm{a}} \leq 1$ (using auxiliary circularly-polarized pump beams) along the same directions $\pm \boldsymbol{e}_{x}$ as the noble-gas spins. We define for each cell the normalized macroscopic alkali-spin operator $\hat{\mathbf{f}}_{i} \equiv M_{\mathrm{a}}^{-1 / 2} \sum_{m=1}^{N_{\mathrm{a}}} \hat{\mathbf{f}}_{i}^{(m)}$, where $M_{\mathrm{a}}=P_{\mathrm{a}} N_{\mathrm{a}}(I+1 / 2)$ is the alkali magnetization, and $I$ is the alkali nuclear spin. Similarly to the noble-gas spins, $\hat{\mathrm{f}}_{i x}$ are considered classical, with $\left\langle\hat{\mathrm{f}}_{i x}\right\rangle= \pm M_{\mathrm{a}}^{1 / 2}$, whereas $\hat{\mathrm{f}}_{i y}$ and $\hat{\mathrm{f}}_{i z}$ are governed by quantum fluctuations. The probe is a square pulse of duration $T$, propagating along $\boldsymbol{e}_{z}$ with initial linear polarization $\boldsymbol{e}_{x}$. We represent its state by the normalized Stokes operators $\hat{\boldsymbol{S}}(z)$ where $\left\langle\hat{S}_{x}\right\rangle^{2}=M_{\mathrm{L}}$ is the total number of photons in the pulse, and $\hat{S}_{y}, \hat{S}_{z}$, describe the ellipticity of the polarization-state subject to quantum polarization-fluctuations.

The Hamiltonian describing the interactions in the system is given by [10, 19]

$$
\mathcal{V}=\hbar J\left(\hat{\mathbf{f}}_{1} \cdot \hat{\mathbf{k}}_{1}+\hat{\mathbf{f}}_{2} \cdot \hat{\mathbf{k}}_{2}\right)+\hbar Q\left(\hat{\mathrm{f}}_{1 z}+\hat{\mathrm{f}}_{2 z}\right) \int \frac{d z^{\prime}}{L} \hat{S}_{z}\left(z^{\prime}\right) .
$$

The first term describes a mutual precession of the alkali and noble-gas spins around each other at a rate $J$. It manifests the coherent collective coupling between these spins via multiple weak spin-exchange collisions [19. The second term in Eq. (2) describes the dispersive interaction of the alkali spins with the far-detuned probe traversing the two cells [11. The spin components along the optical axis $\left(\hat{\mathrm{f}}_{1 z}+\hat{\mathrm{f}}_{2 z}\right)$ govern the Faraday rotation of the light polarization, while circularlypolarized light $\left(\hat{S}_{z}\right)$ acts back to rotate the spins via lightshifts. The coupling rate is given by $Q=(a / T) \sqrt{M_{\mathrm{a}} M_{\mathrm{L}}}$, where $a \propto 1 / \delta_{\mathrm{e}}$ is the unitless optical-coupling coefficient [11, 30 and $L$ is the length of each cell. See Supplementary Material for detailed expressions of $J, Q$, and $a$ 31.

To generate entanglement, we set common precession frequencies $\left(\omega_{\mathrm{a}}, \omega_{\mathrm{b}}\right)$ in the two cells, by tuning the magnetic fields and the light-shifts induced by the pumps in each cell 31. We describe the spin dynamics in a common rotating frame, defined by $\hat{\mathbf{k}}_{i} \rightarrow R_{x}\left(\omega_{\mathrm{b}} t\right) \hat{\mathbf{k}}_{i}$ and $\hat{\mathbf{f}}_{i} \rightarrow R_{x}\left(\omega_{\mathrm{b}} t\right) \hat{\mathbf{f}}_{i}$, where $R_{x}(\theta)$ rotates a vector by an angle $\theta$ around $\boldsymbol{e}_{x}$. In this frame, the alkali spins precess at frequency $\Delta=\omega_{\mathrm{a}}-\omega_{\mathrm{b}}$.

We now take the off-resonance regime $\Delta \gg \gamma_{\mathrm{a}}, J, Q$ and first present the results for negligible relaxations. Given the interaction Hamiltonian (2), we find that the transverse fluctuations $\hat{\mathrm{f}}_{i y}, \hat{\mathrm{f}}_{i z}$ of the alkali spins adia- batically follow the noble-gas spins-fluctuations, and the probe polarization,

$$
\hat{\mathbf{f}}_{i}= \pm \frac{J}{\Delta} \hat{\mathbf{k}}_{i} \pm \frac{Q}{\Delta} \hat{S}_{z} \boldsymbol{e}(t),
$$

where $\boldsymbol{e}(t)=\sin \left(\omega_{\mathrm{b}} t\right) \boldsymbol{e}_{\boldsymbol{y}}+\cos \left(\omega_{\mathrm{b}} t\right) \boldsymbol{e}_{\boldsymbol{z}}$ is the optical axis in the rotating frame. Thus, the large frequency mismatch $\Delta$ renders the interaction dispersive, moderating the response of the alkali spins to both spin-exchange and back-action of light.

We use Eqs. (2 3 to terive the Heisenberg-Langevin equations for the transverse operators $\hat{S}$ and $\hat{\mathbf{k}}_{i}$ [31. First, we find that the difference between the noble-gas spins remains constant

$$
\partial_{t}\left(\hat{\mathbf{k}}_{1}-\hat{\mathbf{k}}_{2}\right)=0 .
$$

Importantly, the preparation of the two cells with oppositely oriented spins eliminates the back-action effect [second term in Eq. (3p] of the probe on the operator $\hat{\mathbf{k}}_{1}-\hat{\mathbf{k}}_{2}$. Second, we find that $\hat{\mathbf{k}}_{1}-\hat{\mathbf{k}}_{2}$ determines the evolution of the probe polarization along the cell

$$
\partial_{z} \hat{S}_{y}=\frac{Q J T}{L \Delta}\left(\hat{\mathbf{k}}_{1}-\hat{\mathbf{k}}_{2}\right) \cdot \boldsymbol{e}(t) .
$$

Equation (5) manifests the indirect Faraday interaction between the probe and the noble-gas spins, with the outgoing polarization $\hat{S}_{y}(L)$ providing a monitor of $\hat{\mathbf{k}}_{1}-\hat{\mathbf{k}}_{2}$. In particular, a simultaneous measurement of the inphase and out-of-phase components of $\hat{S}_{y}(L)$ via homodyne detection yields the nonlocal spin components $\hat{\mathrm{k}}_{1 y}-\hat{\mathrm{k}}_{2 y}$ and $\hat{\mathrm{k}}_{1 z}-\hat{\mathrm{k}}_{2 z}$, respectively.

The procedure for entanglement generation is shown in Fig. 2 Initially, homodyne measurement of the probe, which underwent the evolution in Eq. (5), drives the noble-gas ensembles to a nonclassical two-mode squeezed state, displaced according to the measurement outcome [11. Subsequently, feeding-back the measurement outcome to rotate the spins (using a short magnetic pulse) sets the mean value of their squeezed components to zero, yielding unconditioned entanglement.

To quantify this process, we define canonical operators for the probe $\hat{\boldsymbol{x}}_{\mathrm{L}}(z)=\sqrt{2} \int_{0}^{T} \hat{S}_{y}(z) \boldsymbol{e}(t) d t / T$ and $\hat{\boldsymbol{p}}_{\mathrm{L}}(z)=\sqrt{2} \int_{0}^{T} \hat{S}_{z}(z) \boldsymbol{e}(t) d t / T$, and nonlocal canonical operators for the noble-gas spins $\hat{\boldsymbol{x}}_{\mathrm{b}}(t)=\boldsymbol{e}_{\boldsymbol{x}} \times\left(\hat{\mathbf{k}}_{1}+\right.$ $\left.\hat{\mathbf{k}}_{2}\right) / \sqrt{2}$ and $\hat{\boldsymbol{p}}_{\mathrm{b}}(t)=\left(\hat{\mathbf{k}}_{1}-\hat{\mathbf{k}}_{2}\right) / \sqrt{2}$. These constitute two independent Harmonic oscillators. The total evolution is then given by a set of input-output relations, obtained by integration of Eqs. (4.5) 31.

$$
\begin{aligned}
& \hat{\boldsymbol{x}}_{\mathrm{L}}^{\text {out }}=\hat{\boldsymbol{x}}_{\mathrm{L}}^{\text {in }}+\kappa \hat{\boldsymbol{p}}_{\mathrm{b}}^{\text {in }} \\
& \hat{\boldsymbol{p}}_{\mathrm{b}}^{\text {out }}=\hat{\boldsymbol{p}}_{\mathrm{b}}^{\text {in }} .
\end{aligned}
$$

The input components of the probe $\hat{\boldsymbol{x}}_{\mathrm{L}}^{\text {in }}, \hat{\boldsymbol{p}}_{\mathrm{L}}^{\text {in }}$ comprise the photon shot-noise at $z=0$, and the output components $\hat{\boldsymbol{x}}_{\mathrm{L}}^{\text {out }}, \hat{\boldsymbol{p}}_{\mathrm{L}}^{\text {out }}$ describe the probe state at $z=2 L$ 

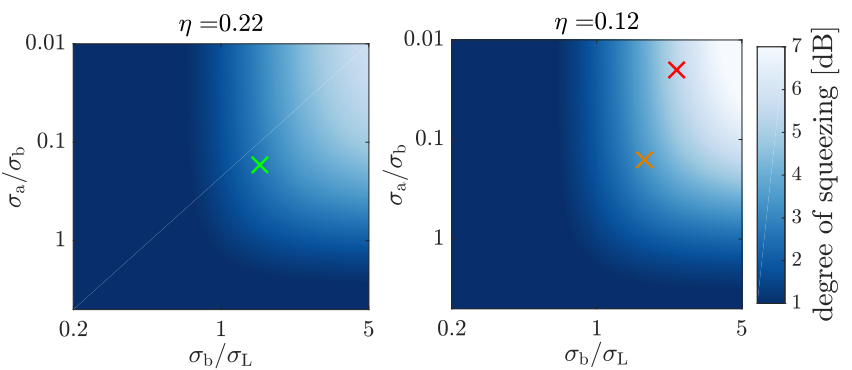

Figure 3. Attainable degree of two-mode spin squeezing for noble-gas ensembles. We present results for both $\eta=0.22$ and $\eta=0.12$, where $\eta$ characterizes the fractional decoherence of the noble-gas spins during the entangling process. The parameters $\sigma_{\mathrm{a}}, \sigma_{\mathrm{b}}$, and $\sigma_{\mathrm{L}}$ denote the contributions of the alkali spin-projection noise, noble-gas spin-projection noise, and photon shot-noise, respectively, to the optical measurements. The squeezing is maximized when the noble-gas noise $\sigma_{\mathrm{b}}$ dominates the measurement. The calculations are done using Eq. (7), with $\sigma_{\mathrm{b}} / \sigma_{\mathrm{L}}=\kappa \sqrt{1-\epsilon}$ and $\sigma_{\mathrm{a}} / \sigma_{\mathrm{b}}=\varrho$. The crosses mark proposed working points with ${ }^{129} \mathrm{Xe}-{ }^{87} \mathrm{Rb}$ (green) and ${ }^{3} \mathrm{He}-\mathrm{K}$ (red,orange).

after the cells. Similarly, the noble-gas spin operators $\hat{\boldsymbol{x}}_{\mathrm{b}}^{\mathrm{in}}, \hat{\boldsymbol{p}}_{\mathrm{b}}^{\text {in }}$ comprise the atomic projection-noise at $t=0$, and $\hat{\boldsymbol{x}}_{\mathrm{b}}^{\text {out }}, \hat{\boldsymbol{p}}_{\mathrm{b}}^{\text {out }}$ describe the collective spin-state at $t=T$. Therefore, Eqs. (6) describe the Faraday rotation $\left(\hat{x}_{\mathrm{L}}^{\text {out }}\right.$ ) of the linearly polarized input light $\left(\hat{\boldsymbol{x}}_{\mathrm{L}}^{\text {in }}\right)$ by the total noble-gas spin $\left(\hat{\boldsymbol{p}}_{\mathrm{b}}^{\mathrm{in}}\right)$, as shown in Fig. 1. (c), with no backaction $\left(\hat{\boldsymbol{p}}_{\mathrm{b}}^{\text {out }}=\hat{\boldsymbol{p}}_{\mathrm{b}}^{\text {in }}\right)$. The unitless coupling constant $\kappa \equiv Q J T / \Delta$ quantifies the net polarization rotation of the probe. It characterizes the measurement strength of the noble-gas spins with respect to the photon shot-noise, depending on the resonant optical-depth of the alkali ensembles [31.

For coherent light and coherent spin-states, the input uncertainties are at the classical minimum, satisfying $\operatorname{var}\left(\hat{x}_{\mathrm{L}, \alpha}^{\mathrm{in}}\right)=\operatorname{var}\left(\hat{p}_{\mathrm{L}, \alpha}^{\text {in }}\right)=\operatorname{var}\left(\hat{x}_{\mathrm{b}, \alpha}^{\text {in }}\right)=\operatorname{var}\left(\hat{p}_{\mathrm{b}, \alpha}^{\text {in }}\right)=1 / 2$ with $\alpha=y, z$. Following the measurement, a magnetic pulse feedback is used for rotating the noble-gas spins from $\hat{\boldsymbol{p}}_{\mathrm{b}}^{\text {out }}=\hat{\boldsymbol{p}}_{\mathrm{b}}^{\text {in }}$ to $\hat{\boldsymbol{p}}_{\mathrm{b}}^{\text {in }}+G \hat{\boldsymbol{x}}_{\mathrm{L}}^{\text {out }}$. The feedback proportionality constant $G$ can be optimally chosen to minimize $\operatorname{var}\left(\hat{p}_{\mathrm{b}, \alpha}^{\text {out }}\right)=\left(2+2 \kappa^{2}\right)^{-1}$ for both $\alpha=y, z$. Identifying $\operatorname{var}\left(\hat{p}_{\mathrm{b}, \alpha}^{\text {out }}\right)=\exp (-2 \xi) / 2$ as the degree of two-mode squeezing, we obtain the squeezing parameter $\xi=\ln \left(1+\kappa^{2}\right) / 2$. Evidently, any system with $\kappa>0$ yields nonzero squeezing and satisfies the inequalities $\operatorname{var}\left(\hat{p}_{\mathrm{b}, \alpha}^{\text {out }}\right)<1 / 2$, thus satisfying the entanglement condition in Eq. (1). We therefore conclude that our scheme correlates the spin-states of two distant noble-gas ensembles, generating unconditional entanglement.

We now return to consider relaxation processes expected in realistic conditions. The mechanisms dominating the relaxation rate $\gamma_{\text {sd }}$ of the alkali spin are absorption of probe photons, collisions with noble-gas atoms, spin destruction during alkali collisions, and collisions with the cell walls [19, 29, 33, 34. Continuous optical- pumping at a rate $R_{\mathrm{op}}$ can be used to maintain a constant alkali magnetization $M_{\mathrm{a}}=P_{\mathrm{a}} N_{\mathrm{a}}(I+1 / 2)$, with $P_{\mathrm{a}}=R_{\mathrm{op}} / \gamma_{\mathrm{a}}$ and $\gamma_{\mathrm{a}}=\gamma_{\mathrm{sd}}+R_{\mathrm{op}}$. The noble gas is hyperpolarized via spin-exchange optical-pumping (SEOP) at a high magnetic field prior to the experiment [29, 35]. For polarized alkali spins, the decoherence rate of the noble-gas spins is $\Gamma_{\mathrm{b}}=\gamma_{\mathrm{b}}+(J / \Delta)^{2} \gamma_{\mathrm{a}}$; it inherits a fraction $(J / \Delta)^{2}$ of the alkali decoherence rate $\gamma_{\mathrm{a}}$, which often dominates $\Gamma_{\mathrm{b}}$ [36]. At low alkali densities, $\gamma_{\mathrm{b}}$ is typically limited by technical magnetic inhomogeneities to $\gamma_{\mathrm{b}} \lesssim(\text { minute })^{-1}$ for ${ }^{129} \mathrm{Xe}$ and $\gamma_{\mathrm{b}} \lesssim(\text { hour })^{-1}$ for ${ }^{3} \mathrm{He}$ [17, 18, 37.

These relaxation processes are accompanied by noise, which increases the measurement variance and limits $\xi$. We generalize Eqs. (6) and include the relaxation and noise effects, deriving the best attainable two-mode squeezing parameter 31]

$$
\xi=\frac{1}{2} \ln \left(\frac{\kappa^{2}(1-\epsilon)(1+\varrho)+1}{\kappa^{2}(1-\epsilon)(\eta+\varrho)+1}\right) .
$$

Here $\epsilon=4 \gamma_{\mathrm{L}} L$ denotes the total fraction of scattered probe photons, $\eta=2 \Gamma_{\mathrm{b}} T$ denotes the fraction of decohered noble-gas spins, and $\varrho=4 q \gamma_{\mathrm{a}} /\left(J^{2} T\right)$ characterizes the ratio between the contributions of alkali spins and noble-gas spins to the projection noise. The unitless parameter $q\left(I, P_{\mathrm{a}}\right) \geq 1$ quantifies the increase of alkali projection-noise (variance) due to imperfect spinpolarization, where $q\left(0, P_{a}\right)=q(I, 1)=1$ [30]. Equation (7) guarantees the generation of entanglement between the two ensembles for $\eta \ll 1$. Notably, it has the same form as for squeezing two alkali ensembles [11] except for the additional parameter $\varrho$. In Fig. 3, we use Eq. (7) to plot the degree of squeezing $\exp (-2 \xi)$ of the two noblegas spin-ensembles as a function of $\kappa \sqrt{1-\epsilon}$ and $\varrho$ for two values of $\eta$.

Our entanglement generation scheme can be realized with various alkali and noble-gas mixtures within a large range of experimental parameters. Here we present a representative configuration for entangling two ${ }^{3} \mathrm{He}$ ensembles in two cylindrical cells of length $L=5 \mathrm{~cm}$ and cross-section $A=2 \mathrm{~mm}^{2}$. We consider a gaseous mixture of 880 Torr ${ }^{3} \mathrm{He}, 70$ Torr $\mathrm{N}_{2}$, and a droplet of $\mathrm{K}$ at $250^{\circ} \mathrm{C}$. Here $R_{\mathrm{op}}=1.6 \gamma_{\mathrm{a}}$ yields $P_{\mathrm{a}}=0.62$ [with $q\left(3 / 2, P_{\mathrm{a}}\right)=$ $1.22]$ and $P_{\mathrm{b}}=0.56$, assuming $\gamma_{\mathrm{b}}^{-1}=50$ hour. The 400$\mathrm{mW}$ probe is detuned $3 \mathrm{THz}$ from the optical line, and $B_{1} \approx 10 \mathrm{mG}$. Homodyne detection for $T=200 \mathrm{msec}$ yields $\kappa=2, \epsilon=0.3, \eta=0.125$, and $\varrho=0.162$, generating $4 \mathrm{~dB}$ of two-mode squeezing $(\xi=0.45)$, which could live for tens of hours. The performance for this configuration is marked in Fig. 3 (orange cross). Other exemplary experimental configurations, marked in Fig. 3 and detailed in 31, yield $6 \mathrm{~dB}$ of squeezing for ${ }^{3} \mathrm{He}-\mathrm{K}$ mixture (red cross) and $3 \mathrm{~dB}$ of squeezing for ${ }^{129} \mathrm{Xe}^{87} \mathrm{Rb}$ mixture (green cross).

The long coherence time within each noble-gas spin ensemble ideally also applies to the entanglement life- 
time, even though each ensemble comprises a macroscopic number of spins. In the Holstein-Primakoff approximation, the number of spin excitations is independent of the total number of spins. Indeed we show in 31 that the squeezed quadrature, $\operatorname{var}\left(\hat{\boldsymbol{p}}_{\mathrm{b}}^{\text {out }}\right)<1 / 2$, decays at a constant rate $2 \Gamma_{\mathrm{b}}$.

The long-lived entanglement can be verified by applying an off-resonant probe pulse, measuring the two spin-ensembles simultaneously by utilizing the same experimental configuration used for their generation [10]. Alternatively, the spin of each cell could be measured independently, and their cross-correlations can be found. In systems featuring strong-coupling between the alkali and noble-gas $\left(J \gg \gamma_{\mathrm{a}}\right)$. Transfer times $J^{-1}$ of a few milliseconds are possible [19], realizing fast operations yet maintaining long coherence-times. The alkali squeezedstate could then be projected using a short probe pulse.

In summary, we presented a scheme for entangling the collective nuclear spins of two macroscopic noble-gas ensembles, relying on alkali spin for obtaining an indirect Faraday interaction between the noble-gas and light. The role of relaxations has been considered, revealing that sizable degree of entanglement can be generated at standard experimental conditions, and maintained for extremely long times. With technologically available miniature cells [38 40] and exceptionally long coherence-times, entanglement of hot spin ensembles holds a promise for realizing new quantum-optics applications and enhanced sensing at ambient conditions. The scheme could potentially be extended to generate entanglement in other physical systems having hybrid electronic and opticallyinaccessible nuclear spins, including quantum dots, diamond color-centers, and rare-earth impurities interacting with nearby nuclear spins in the crystal.

We acknowledge financial support by the European Research Council starting investigator grant Q-PHOTONICS 678674, ERC Advanced Grant QUANTUM-N, the Israel Science Foundation and ICORE, the Pazy Foundation, the Minerva Foundation with funding from the Federal German Ministry for Education and Research, and the Laboratory in Memory of Leon and Blacky Broder. ESP was supported by VILLUM FONDEN under a Villum Investigator Grant, grant no. 25880

* Corresponding author or.katz@weizmann.ac.il

[1] S. Haroche, Phys. Today 36-42 (July 1998).

[2] R. Horodecki, P. Horodecki, M. Horodecki and K. Horodecki, Rev. Mod. Phys. 81, 865 (2009)

[3] M. A. Nielsen \& I. L. Chuang, Quantum Computation and Quantum Information (Cambridge Univ. Press, Cambridge, UK, 2000).

[4] T. D. Ladd, F. Jelezko, R. Laflamme, Y. Nakamura, C. Monroe and J. L. O'Brien . Nature 464, 45-53 (2010).
[5] S. L. Braunstein and P. van Loock. Rev. Mod. Phys. 77, 513-577 (2005).

[6] N. Gisin \& R. Thew. Nature Photon. 1, 165-171 (2007).

[7] H. J. Kimble. Nature 453, 1023-1030 (2008).

[8] L. Pezzé, A. Smerzi, M. K. Oberthaler, R. Schmied \& P. Treutlein. Rev. Mod. Phys. 90, 035005 (2018).

[9] W. Wasilewski, K. Jensen, H. Krauter, J. J. Renema, M. V. Balabas \& E.S. Polzik. Phys. Rev. Lett. 104, 133601 (2010).

[10] B. Julsgaard, A. Kozhekin \& E. S. Polzik. Nature 413, 400-403 (2001).

[11] K. Hammerer, A. S. Sørensen, \& E. S. Polzik, Rev. Mod. Phys. 82, 1041-1093 (2010).

[12] J. Kong, R. Jiménez-Martínez, C. Troullinou, V. G. Lucivero \& M. W. Mitchell. arXiv preprint arXiv:1804.07818 (2018).

[13] H. Häffner, et al. Nature 438, 643-646 (2005).

[14] I. Bloch. Nature 453, 1016-1022 (2008).

[15] P. Neumann, N. Mizuochi, F. Rempp, P. Hemmer, H. Watanabe, S. Yamasaki, V. Jacques, T. Gaebel, F. Jelezko, J. Wrachtrup. Science 320, 1326-1329 (2008).

[16] A. D. O'Connell, et al. Nature 464, 697-703 (2010).

[17] C. Gemmel, et al. Eur. Phys. J. D 57, 303 (2010).

[18] T. R. Gentile, P. J. Nacher, B. Saam \& T. G. Walker. Rev. Mod. Phys. 89, 045004 (2017).

[19] O. Katz, R. Shaham \& O. Firstenberg. arXiv preprint arXiv:1905.12532 (2019).

[20] A. Dantan, G. Reinaudi, A. Sinatra, F. Laloë, E. Giacobino \& M. Pinard. Phys. Rev. Lett. 95, 123002 (2005).

[21] A. Kuzmich, K. Mölmer \& E. S. Polzik. Phys. Rev. Lett. 79, 4782 (1997).

[22] A. Kuzmich, L. Mandel \& N. P. Bigelow, Phys. Rev. Lett. 85, 1594-1597 (2000).

[23] A. Kuzmich \& E. S. Polzik. Phys. Rev. Lett. 85, 5639-5642 (2000).

[24] L. M. Duan, J. I. Cirac, P. Zoller \& E. S. Polzik. Phys. Rev. Lett. 85, 5643-5646 (2000).

[25] C. B. Møller, R. A. Thomas, G. Vasilakis, E. Zeuthen, Y. Tsaturyan, K. Jensen, A. Schliesser, K. Hammerer \& E. S. Polzik. Nature 547, 191-195 (2017).

[26] E. Zeuthen, E. S. Polzik \& F. Y. Khalili. Phys. Rev. D 100, 062004 (2019).

[27] G. Reinaudi , A. Sinatra , A. Dantan \& M. Pinard. Squeezing and entangling nuclear spins in helium 3. Journal of Modern Optics 54675 (2007).

[28] L. M. Duan, G. Giedke, J. I. Cirac \& P. Zoller. Phys. Rev. Lett. 84, 2722-2725 (2000).

[29] W. Happer, Y. Y. Jau \& T. Walker. Optically Pumped Atoms. 159-218 (WILEY-VCH Press, 2010).

[30] G. Vasilakis, V. Shah \& M. V. Romalis. Phys. Rev. Lett. 106, 143601 (2011).

[31] See Supplemental Material [url] for the derivation of the equations of motion, analysis of the entanglement lifetime, presentation of additional experimental configurations, and derivation of optical depth as a resource for entanglement generation, which includes Ref. 32].

[32] S. M. Rochester, D. S. Hsiung, D. Budker, R. Y. Chiao, D. F. Kimball, and V. V. Yashchuk, Phys. Rev. A 63, 043814 (2001).

[33] O. Katz, O. Peleg \& O. Firstenberg. Phys. Rev. Lett. 115, 113003 (2015).

[34] O. Katz \& O. Firstenberg. Nat. Commun. 9, 2074 (2018).

[35] W. Happer, E. Miron, S. Schaefer, D. Schreiber, W. A. van Wijngaarden \& X. Zeng. Phys. Rev. A 29, 3092-3110 
(1984).

[36] T. W. Kornack \& M. V. Romalis. Phys. Rev. Lett. 89, 253002 (2002).

[37] X. Zeng, Z. Wu, T. Call, E. Miron, D. Schreiber \& W. Happer. Phys. Rev. A 31, 260 (1985).

[38] M. E. Limes, N. Dural, M. V. Romalis, E. L. Foley, T. W. Kornack, A. Nelson, L. R. Grisham \& J. Vaara. Phys. Rev. A 100, 010501(R) (2019).

[39] T. G. Walker \& M. S. Larsen. Adv. At. Mol. Opt. Phy. 65, 373-401. (2016).

[40] D. A. Thrasher, S. S. Sorensen and T. G. Walker, arXiv preprint arXiv:1912.04991 (2019). 


\section{Supplementary Information for "Long-lived entanglement generation of nuclear spins using coherent light"}

\section{Appendix A: Derivation of the equations of motion}

To derive the equations of motion of the spin and light operators, we consider the total atomic Hamiltonian $H_{\text {tot }}=\mathcal{H}_{1}+\mathcal{H}_{2}+\mathcal{V}$, composed of the interaction Hamiltonian $\mathcal{V}$ [given in Eq. (2) in the main text] and the bare atomic Hamiltonians of the two cells $\mathcal{H}_{1}$ and $\mathcal{H}_{2}$. The bare Hamiltonians depend on the hyperfine interaction within each alkali atom and on the energy splitting of the spin levels. They are given by $(i=1,2)$

$$
\mathcal{H}_{i}=\sum_{n=1}^{N_{\mathrm{a}}}\left(A_{\mathrm{hpf}} \hat{\boldsymbol{i}}_{i}^{(n)} \cdot \hat{\boldsymbol{s}}_{i}^{(n)}+\hbar \tilde{\omega}_{i \mathrm{a}} \hat{s}_{i x}^{(n)}\right)+\sum_{n=1}^{N_{\mathrm{b}}} \hbar \tilde{\omega}_{i \mathrm{~b}} \hat{k}_{i x}^{(n)}
$$

In both cells $(i=1,2)$, each alkali atom denoted by $m$ has a spin $\hat{\mathbf{f}}_{i}^{(m)}=\hat{\boldsymbol{s}}_{i}^{(m)}+\hat{\mathbf{i}}_{i}^{(m)}$, composed of an electronic spin-1/2 operator $\hat{\boldsymbol{s}}_{i}^{(m)}$ and a nuclear spin $I>0$ operator $\hat{\mathbf{i}}_{i}^{(m)}$, coupled by the strong hyperfine interaction [S29]. $A_{\mathrm{hpf}}$ denotes the hyperfine coupling constant, $\tilde{\omega}_{i \mathrm{a}}=g_{a} B_{i}+\Omega_{i}$ are the energy splittings of the alkali spins and $\tilde{\omega}_{i \mathrm{~b}}=g_{\mathrm{b}} B_{i}$ are the level splittings of the noble-gas spins. Here $g_{\mathrm{a}}$ and $g_{\mathrm{b}}$ denote the gyromagnetic ratios of the polarized alkali and noble-gas spins respectively, where the gyromagnetic ratio of the alkali spins $g_{\mathrm{a}}$ is $100-1000$ times larger than that of the noble-gas spins $g_{\mathrm{b}} . B_{i} \boldsymbol{e}_{x}$ are the applied magnetic fields in both cells (introducing the Larmor-precession rates $g_{\mathrm{a}} B_{i}$ and $\left.g_{\mathrm{b}} B_{i}\right)$ and $\Omega_{i}$ denote light shifts induced by the pump beams on the alkali spins. The hyperfine interaction is the dominant interaction in the Hamiltonian, and therefore for time scales longer than $1 / A_{\mathrm{hpf}}$ and high polarization of the alkali spin ensemble, we can identify $\hat{\boldsymbol{s}}^{(n)} \equiv[I]^{-1} \hat{\mathbf{f}}^{(n)}$, where $[I]=2 I+1$.

For alkali-noble-gas mixtures polarized along $\pm \boldsymbol{e}_{x}$, the collisional interaction leads to coherent exchange between the quantum spin fluctuations (the transverse spin components), as well as to fictitious magnetic fields along $\pm \boldsymbol{e}_{x}$ imposed by each species on the other. Consequently, the total precession frequencies of the fluctuations of the alkali and noble-gas spins are given respectively by $\omega_{i \mathrm{a}}=\tilde{\omega}_{i \mathrm{a}} \pm J \sqrt{M_{\mathrm{b}} / M_{\mathrm{a}}}$ and $\omega_{i \mathrm{~b}}=\tilde{\omega}_{i \mathrm{~b}} \pm J \sqrt{M_{\mathrm{a}} / M_{\mathrm{b}}}$. To synchronize the precession frequencies in both cells, we set $B_{2}$ and $\left(\Omega_{2}-\Omega_{1}\right)$ to satisfy $\omega_{1 \mathrm{a}}=\omega_{2 \mathrm{a}} \equiv \omega_{\mathrm{a}}$ and $\omega_{1 \mathrm{~b}}=\omega_{2 \mathrm{~b}} \equiv \omega_{\mathrm{b}}$, for any choice of $B_{1}$. Under these conditions, the quantum dynamics of the system derived from the Hamiltonian $H_{\text {tot }}$ is described by the Heisenberg-Langevin equations for the transverse operators

$$
\begin{aligned}
\partial_{z} \hat{\boldsymbol{S}} & =\frac{T Q}{L}\left(\hat{\mathrm{f}}_{1 z}+\hat{\mathrm{f}}_{2 z}\right) \boldsymbol{e}_{y}-\gamma_{\mathrm{L}} \hat{\boldsymbol{S}}+\hat{\boldsymbol{F}}_{\mathrm{L}} \\
\partial_{t} \hat{\mathbf{f}}_{i} & =\left( \pm J \hat{\mathbf{k}}_{i}-\omega_{\mathrm{a}} \hat{\mathbf{f}}_{i}\right) \times \boldsymbol{e}_{x} \pm Q \hat{S}_{z} \boldsymbol{e}_{y}-\gamma_{\mathrm{a}} \hat{\mathbf{f}}_{i}+\hat{\boldsymbol{F}}_{i \mathrm{a}} \\
\partial_{t} \hat{\mathbf{k}}_{i} & =\left( \pm J \hat{\mathbf{f}}_{i}-\omega_{\mathrm{b}} \hat{\mathbf{k}}_{i}\right) \times \boldsymbol{e}_{x}-\gamma_{\mathrm{b}} \hat{\mathbf{k}}_{i}+\hat{\boldsymbol{F}}_{i \mathrm{~b}}
\end{aligned}
$$

Here $\gamma_{\mathrm{L}}$ denotes the attenuation per-unit-length of the probe (including the absorption by the alkali atoms), $\gamma_{\mathrm{a}}$ denotes the total decoherence rate of the alkali spins in the presence of the probe, and $\gamma_{\mathrm{b}}$ denotes the slow relaxation of the noble-gas spins [S24. The vacuum noise operators $\hat{\boldsymbol{F}}_{\mathrm{L}}, \hat{\boldsymbol{F}}_{i \mathrm{a}}$, and $\hat{\boldsymbol{F}}_{i \mathrm{~b}}$ are associated with these decays [S11, S19]. The spin-exchange interaction allows for coherent state-exchange between the alkali and noble-gas spins within each of the two cells independently at a rate $J=g \sqrt{M_{\mathrm{a}} M_{\mathrm{b}}} /(A L)$. The coherent spin-exchange rate coefficient is $g$, with $g=4.9 \times 10^{-15} \mathrm{~cm}^{3} \mathrm{~s}^{-1}$ for a $\mathrm{K}-{ }^{3} \mathrm{He}$ mixture or $g=1.9 \times 10^{-13} \mathrm{~cm}^{3} \mathrm{~s}^{-1}$ for ${ }^{87} \mathrm{Rb}-{ }^{129} \mathrm{Xe}$ [S19, S29]. At the same time, the polarization state of the probe is altered by both ensembles together: $\hat{S}_{y}$ depends on the nonlocal spin operator $\hat{\mathrm{f}}_{1 z}+\hat{\mathrm{f}}_{2 z}$, and $\hat{S}_{z}$ exerts a common back-action light-shift on the two cells. The optical coupling rate $Q=(a / T) \sqrt{M_{\mathrm{a}} M_{\mathrm{L}}}$ depends on $a=2 r_{\mathrm{e}} c f /\left[A \delta_{\mathrm{e}}(2 I+1)\right]$, where $r_{\mathrm{e}}=2.8 \times 10^{-17} \mathrm{~cm}$ is the classical electron radius, $f \leq 1$ is the oscillator strength of the atomic transition, and $\delta_{\mathrm{e}}$ is the detuning of the laser from the optical transition. Also note that the operators in Eqs. S2 S4 satisfy the commutation relations $\left[\hat{\mathrm{f}}_{i y}, \hat{\mathrm{f}}_{j z}\right]= \pm i \delta_{i j}$ for the alkali, $\left[\hat{S}_{y}\left(z^{\prime}\right), \hat{S}_{z}\left(z^{\prime \prime}\right)\right]=i c T \delta\left(z^{\prime}-z^{\prime \prime}\right)$ for the light and $\left[\hat{\mathrm{k}}_{i y}, \hat{\mathrm{k}}_{j z}\right]= \pm i \delta_{i j}$ for the noble-gas spins.

To simplify Eqs. (S2 S4), we transform the system to the rotating frame of the noble-gas spins and describe the adiabatic following of the alkali in the limit of large magnetic field limit (the off-resonance regime) $\Delta \gg \gamma_{\mathrm{a}}, J, Q$. The formal transformation of the collective spin operators in each cell to the rotating frame is given by $\hat{\boldsymbol{f}}_{i}^{\prime}=R_{x}\left(\omega_{\mathrm{b}}\right) \hat{\boldsymbol{f}}_{i}$ 
and $\hat{\boldsymbol{k}}_{i}^{\prime}=R_{x}\left(\omega_{\mathrm{b}}\right) \hat{\boldsymbol{k}}_{i}$, using the standard rotation matrix

$$
R_{x}\left(\omega_{\mathrm{b}}\right)=\left(\begin{array}{ccc}
1 & 0 & 0 \\
0 & \cos \left(\omega_{\mathrm{b}} t\right) & \sin \left(\omega_{\mathrm{b}} t\right) \\
0 & -\sin \left(\omega_{\mathrm{b}} t\right) & \cos \left(\omega_{\mathrm{b}} t\right)
\end{array}\right) .
$$

The operators $\hat{\boldsymbol{f}}^{\prime}$ and $\hat{\boldsymbol{k}}^{\prime}$ are the stationary spin components of the alkali and noble-gas spins, respectively. The dynamics of the $y, z$ components of the alkali spins in the rotating frame is then given by

$$
\partial_{t} \hat{\mathbf{f}}_{i}^{\prime}=\left( \pm J \hat{\mathbf{k}}_{i}^{\prime}-\Delta \hat{\mathbf{f}}_{i}^{\prime}\right) \times \boldsymbol{e}_{x} \pm Q \hat{S}_{z} \boldsymbol{e}_{y}(t)-\gamma_{\mathrm{a}} \hat{\mathbf{f}}_{i}^{\prime}+\hat{\boldsymbol{F}}_{i \mathrm{a}}^{\prime}
$$

We are interested in the slow, adiabatic dynamics of $\hat{\mathbf{f}}_{i}^{\prime}$, which naturally oscillates at a rate $\Delta$. The leading order of the dynamics is thus determined by considering the instantaneous steady state $\partial_{t} \hat{\mathbf{f}}_{i}^{\prime}=0$, which yields the linear relation

$$
\hat{\mathbf{f}}_{i}^{\prime}=\frac{ \pm 1}{\sqrt{\Delta^{2}+\gamma_{\mathrm{a}}^{2}}} R_{x}(\psi) \cdot\left(Q \hat{S}_{z} \boldsymbol{e}(t)+J \hat{\mathbf{k}}_{i}^{\prime}+\hat{\boldsymbol{F}}_{i \mathrm{a}}^{\prime}\right) .
$$

Here $\boldsymbol{e}(t)=\sin \left(\omega_{\mathrm{b}} t\right) \boldsymbol{e}_{\boldsymbol{y}}+\cos \left(\omega_{\mathrm{b}} t\right) \boldsymbol{e}_{\boldsymbol{z}}$ is the optical axis in the rotating frame, and we define $\cos \psi \equiv \Delta / \sqrt{\Delta^{2}+\gamma_{\mathrm{a}}^{2}}$ and $\sin \psi \equiv \gamma_{\mathrm{a}} / \sqrt{\Delta^{2}+\gamma_{\mathrm{a}}^{2}}$. Eq. S7 describes the slow temporal dependence of the alkali spin operators on the noble-gas spins via spin-exchange, on the light circular polarization via back-action noise, and on the infiltrated vacuum white noise associated with the decay rate $\gamma_{\mathrm{a}}$. The noise terms are given by $\hat{\boldsymbol{F}}_{i a}^{\prime}=R_{x}\left(\omega_{\mathrm{b}}\right) \hat{\boldsymbol{F}}_{i a}$, which are statistically equivalent to $\hat{\boldsymbol{F}}_{i a}$. In the off-resonance regime $\Delta \gg \gamma_{\mathrm{a}}$, we obtain $\psi \ll 1$, such that the leading term in Eq. (S7) is free of decay and noise, yielding the simple form of Eq. (3) in the main text (note that in the main text, we dropped the prime notation for brevity).

We now substitute Eq. S7 in Eqs. S2 and S4 to obtain the dynamics of $\hat{\boldsymbol{S}}$ as a function of $\hat{\mathbf{k}}_{i}^{\prime}$

$$
\begin{aligned}
\partial_{z} & \hat{\boldsymbol{S}}=\frac{T Q}{L}\left(\cos \left(\omega_{\mathrm{b}} t\right)\left(\hat{\mathrm{f}}_{1 z}^{\prime}+\hat{\mathrm{f}}_{2 z}^{\prime}\right)+\sin \left(\omega_{\mathrm{b}} t\right)\left(\hat{\mathrm{f}}_{1 y}^{\prime}+\hat{\mathrm{f}}_{2 y}^{\prime}\right)\right) \boldsymbol{e}_{y}-\gamma_{\mathrm{L}} \hat{\boldsymbol{S}}+\hat{\boldsymbol{F}}_{\mathrm{L}} \\
= & \frac{1}{L} \frac{T Q}{\sqrt{\Delta^{2}+\gamma_{\mathrm{a}}^{2}}} \sum_{i=1}^{2}(-1)^{i+1}\left[\left(Q \hat{S}_{z}+J \hat{\mathrm{k}}_{i z}+\hat{F}_{i \mathrm{a}, \mathrm{y}}\right) \cos \psi-\left(J \hat{\mathrm{k}}_{i y}+\hat{F}_{i \mathrm{a}, \mathrm{z}}\right) \sin \psi\right] \boldsymbol{e}_{y}-\gamma_{\mathrm{L}} \hat{\boldsymbol{S}}+\hat{\boldsymbol{F}}_{\mathrm{L}} \\
= & \frac{1}{L} \frac{T Q}{\sqrt{\Delta^{2}+\gamma_{\mathrm{a}}^{2}}}\left[J\left(\cos \psi\left(\hat{\mathrm{k}}_{1 z}-\hat{\mathrm{k}}_{2 z}\right)-\sin \psi\left(\hat{\mathrm{k}}_{1 y}-\hat{\mathrm{k}}_{2 y}\right)\right)+\sum_{i=1}^{2}(-1)^{i+1}\left(\cos \psi \hat{F}_{i \mathrm{a}, \mathrm{y}}+\sin \psi \hat{F}_{i \mathrm{a}, \mathrm{z}}\right)\right] \boldsymbol{e}_{y}-\gamma_{\mathrm{L}} \hat{\boldsymbol{S}}+\hat{\boldsymbol{F}}_{\mathrm{L}} \\
= & \frac{1}{L} \frac{T Q}{\sqrt{\Delta^{2}+\gamma_{\mathrm{a}}^{2}}}\left[J\left(\sin \left(\omega_{\mathrm{b}} t-\psi\right)\left(\hat{\mathrm{k}}_{1 y}^{\prime}-\hat{\mathrm{k}}_{2 y}^{\prime}\right)+\cos \left(\omega_{\mathrm{b}} t-\psi\right)\left(\hat{\mathrm{k}}_{1 z}^{\prime}-\hat{\mathrm{k}}_{2 z}^{\prime}\right)\right)+\sqrt{2} \hat{F}_{\mathrm{a}, \mathrm{y}}\right] \boldsymbol{e}_{y}-\gamma_{\mathrm{L}} \hat{\boldsymbol{S}}+\hat{\boldsymbol{F}}_{\mathrm{L}}
\end{aligned}
$$

We first note, when moving from Eq. $(\mathrm{S} 9)$ to Eq. S10, that the dependence of $\hat{S}_{y}$ on $\hat{S}_{z}$ - also known as polarization selfrotation [S32- is canceled by using the double cell configuration with opposite alkali spin polarization. In Eq. (S11), we use the identity $R_{x}\left(\omega_{\mathrm{b}} t\right) R_{x}(-\psi)=R_{x}\left(\omega_{\mathrm{b}} t-\psi\right)$ and identify the noise process $\hat{F}_{\mathrm{a}, \mathrm{y}} \equiv \sum_{i=1}^{2}(-1)^{i+1}\left(\cos \psi \hat{F}_{i \mathrm{a}, \mathrm{y}}+\right.$ $\left.\sin \psi \hat{F}_{i \mathrm{a}, \mathrm{z}}\right) / \sqrt{2}$, which has identical statistics as $\hat{F}_{1 \mathrm{a}, \mathrm{y}}$ and $\hat{F}_{1 \mathrm{a}, \mathrm{z}}$. For negligible loss mechanisms $\psi, \gamma_{\mathrm{L}} L \ll 1$, Eq. (S11) provides Eq. (5) in the main text. We also note that in the absence of loss, $S_{z}$ remains constant throughout the cell.

Similarly, we derive the equations for the noble-gas spins in each cell in the rotating frame

$$
\begin{aligned}
& \partial_{t} \hat{\mathbf{k}}_{i}^{\prime}= \pm J R_{x}\left(\frac{\pi}{2}\right) \hat{\mathbf{f}}_{i}^{\prime}-\gamma_{\mathrm{b}} \hat{\mathbf{k}}_{i}^{\prime}+\hat{\boldsymbol{F}}_{i \mathrm{~b}} \\
& \quad=\frac{J}{\sqrt{\Delta^{2}+\gamma_{\mathrm{a}}^{2}}} R_{x}(\psi) R_{x}\left(\frac{\pi}{2}\right) \cdot\left(Q \hat{S}_{z} \boldsymbol{e}(t)+J \hat{\mathbf{k}}_{i}^{\prime}+\hat{\boldsymbol{F}}_{i \mathrm{a}}^{\prime}\right)-\gamma_{\mathrm{b}} \hat{\mathbf{k}}_{i}^{\prime}+\hat{\boldsymbol{F}}_{i \mathrm{~b}} \\
& =\frac{J Q}{\sqrt{\Delta^{2}+\gamma_{\mathrm{a}}^{2}}} \hat{S}_{z}\left(\cos \left(\omega_{\mathrm{b}} t+\psi\right) \boldsymbol{e}_{y}-\cos \left(\omega_{\mathrm{b}} t+\psi\right) \boldsymbol{e}_{z}\right)+\delta \omega_{\mathrm{b}} \hat{\mathbf{k}}_{i}^{\prime} \times \boldsymbol{e}_{x}-\Gamma_{\mathrm{b}} \hat{\mathbf{k}}_{i}^{\prime}+\hat{\boldsymbol{F}}_{i \mathrm{~b}}^{\prime}
\end{aligned}
$$

The first term describes the back-action of the probe circular polarization on the noble-gas spins, mediated via its effect on the alkali. Interestingly, the back-action is the same in both cells, being polarized in opposite orientations. 
The second term, with $\delta \omega_{\mathrm{b}}=\Delta J^{2} /\left(\Delta^{2}+\gamma_{\mathrm{a}}^{2}\right)$, describes a small spin-exchange induced shift, typically satisfying $\delta \omega_{\mathrm{b}} \ll \omega_{\mathrm{b}}$. This small shift can be taken into account by the simple transformation $\omega_{\mathrm{b}} \rightarrow \omega_{\mathrm{b}}-\delta \omega_{\mathrm{b}}$ in the equations. The third term describes the decoherence of the noble-gas spins with the total rate $\Gamma_{\mathrm{b}}=\gamma_{\mathrm{b}}+\gamma_{\mathrm{a}} J^{2} /\left(\Delta^{2}+\gamma_{\mathrm{a}}^{2}\right)$, which includes the alkali-induced relaxation as described in the main text. $\hat{\boldsymbol{F}}_{i \mathrm{~b}}^{\prime}$ denotes a quantum white noise operator associated with the decay rate $\Gamma_{\mathrm{b}}$.

We now consider the dynamics of the nonlocal noble-gas spin operators. For the difference of the spin operators $\hat{\mathbf{k}}_{1}^{\prime}-\hat{\mathbf{k}}_{2}^{\prime}$, we find

$$
\partial_{t}\left(\hat{\mathbf{k}}_{1}^{\prime}-\hat{\mathbf{k}}_{2}^{\prime}\right)=-\Gamma_{\mathrm{b}}\left(\hat{\mathbf{k}}_{1}^{\prime}-\hat{\mathbf{k}}_{2}^{\prime}\right)+\sqrt{2} \hat{\boldsymbol{F}}_{\mathrm{b}-},
$$

where we define $\hat{\boldsymbol{F}}_{\mathrm{b} \pm} \equiv\left(\hat{\boldsymbol{F}}_{1 b}^{\prime} \pm \hat{\boldsymbol{F}}_{2 \mathrm{~b}}^{\prime}\right) \sqrt{2}$. Here the common probe back-action is canceled, in accordance with the requirements of the EPR entanglement criterion [cf. Eq. (1) in the main text]. For low noble-gas losses $\Gamma_{\mathrm{b}} T \ll 1$, Eq. (S15) becomes Eq. (4) in the main text. The sum of spin operators, is significantly affected by the probe backaction, given by

$$
\partial_{t}\left(\hat{\mathbf{k}}_{1}^{\prime}+\hat{\mathbf{k}}_{2}^{\prime}\right)=\frac{2 J Q}{\sqrt{\Delta^{2}+\gamma_{\mathrm{a}}^{2}}} \hat{S}_{z}\left(\cos \left(\omega_{\mathrm{b}} t+\psi\right) \boldsymbol{e}_{y}-\cos \left(\omega_{\mathrm{b}} t+\psi\right) \boldsymbol{e}_{z}\right)-\Gamma_{\mathrm{b}}\left(\hat{\mathbf{k}}_{1}^{\prime}+\hat{\mathbf{k}}_{2}^{\prime}\right)+\sqrt{2} \hat{\boldsymbol{F}}_{\mathrm{b}+} \cdot
$$

Before solving Eqs. (S11) and (S15, S16), we renormalize the operators and transform them to the canonical form [S11]. Using the definitions in the main text, we find two independent sets of harmonic oscillator operators, which we here explicitly identify. The first set describes the in-phase component of the light polarization operators

$$
\begin{aligned}
& \hat{x}_{\mathrm{L}, y}(z)=\frac{\sqrt{2}}{T} \int_{0}^{T} \hat{S}_{y}(z) \sin \left(\omega_{\mathrm{b}} t\right) d t, \\
& \hat{p}_{\mathrm{L}, y}(z)=\frac{\sqrt{2}}{T} \int_{0}^{T} \hat{S}_{z}(z) \sin \left(\omega_{\mathrm{b}} t\right) d t,
\end{aligned}
$$

coupled to the spin operators

$$
\begin{aligned}
& \hat{x}_{\mathrm{b}, y}(t)=-\frac{1}{\sqrt{2}}\left(\hat{\mathrm{k}}_{1 z}^{\prime}+\hat{\mathrm{k}}_{2 z}^{\prime}\right), \\
& \hat{p}_{\mathrm{b}, y}(t)=\frac{1}{\sqrt{2}}\left(\hat{\mathrm{k}}_{1 y}^{\prime}-\hat{\mathrm{k}}_{2 y}^{\prime}\right) .
\end{aligned}
$$

The second set is given by the operators

$$
\begin{aligned}
& \hat{x}_{\mathrm{L}, z}(z)=\frac{\sqrt{2}}{T} \int_{0}^{T} \hat{S}_{y}(z) \cos \left(\omega_{\mathrm{b}} t\right) d t \\
& \hat{p}_{\mathrm{L}, z}(z)=\frac{\sqrt{2}}{T} \int_{0}^{T} \hat{S}_{z}(z) \cos \left(\omega_{\mathrm{b}} t\right) d t \\
& \hat{x}_{\mathrm{b}, z}(t)=\frac{1}{\sqrt{2}}\left(\hat{\mathrm{k}}_{1 y}^{\prime}+\hat{\mathrm{k}}_{2 y}^{\prime}\right) \\
& \hat{p}_{\mathrm{b}, z}(t)=\frac{1}{\sqrt{2}}\left(\hat{\mathrm{k}}_{1 z}^{\prime}-\hat{\mathrm{k}}_{2 z}^{\prime}\right) .
\end{aligned}
$$

The light vector operators consist of two sets of standard Harmonic oscillator operators satisfying $\left[\hat{x}_{\mathrm{L}, \alpha}, \hat{p}_{\mathrm{L}, \beta}\right]=i \delta_{\alpha \beta}$ for $\alpha, \beta \in\{y, z\}$. Similarly, the atomic operators satisfy $\left[\hat{x}_{\mathrm{b}, \alpha}, \hat{p}_{\mathrm{b}, \beta}\right]=i \delta_{\alpha \beta}$.

We first derive the leading terms in the input and output relations of the system, absent relaxation and noise. Substituting the definitions (S17, S24) into Eq. (S11) and spatially integrating along the beam path yield the light response to the emerging Faraday interaction with noble-gas spins

$$
\begin{aligned}
& \hat{\boldsymbol{x}}_{\mathrm{L}}^{\text {out }}=\hat{\boldsymbol{x}}_{\mathrm{L}}^{\text {in }}+\kappa \hat{\boldsymbol{p}}_{\mathrm{b}}^{\text {in }}, \\
& \hat{\boldsymbol{p}}_{\mathrm{L}}^{\text {out }}=\hat{\boldsymbol{p}}_{\mathrm{L}}^{\text {in }},
\end{aligned}
$$

where

$$
\kappa=\frac{J Q T}{\sqrt{\Delta^{2}+\gamma_{\mathrm{a}}^{2}}}
$$


is the unitless optical coupling strength. Similarly, substituting the definitions (S17) S24 in Eqs. (S15) S16) and temporally integrating for the pulse duration $T$ yield the atomic evolution of the spins by the emerging Faraday interaction with noble-gas spins

$$
\begin{aligned}
& \hat{\boldsymbol{x}}_{\mathrm{b}}^{\text {out }}=\hat{\boldsymbol{x}}_{\mathrm{b}}^{\text {in }}+\kappa \hat{\boldsymbol{p}}_{\mathrm{L}}^{\text {in }}, \\
& \hat{\boldsymbol{p}}_{\mathrm{b}}^{\text {out }}=\hat{\boldsymbol{p}}_{\mathrm{b}}^{\text {in }} .
\end{aligned}
$$

Equations. S25 and S29 give Eqs. (6) in the main text. In the presence of noise and relaxations, the modified input-output relations are given by

$$
\begin{aligned}
& \hat{\boldsymbol{x}}_{\mathrm{L}}^{\text {out }}=\sqrt{1-\epsilon}\left(\hat{\boldsymbol{x}}_{\mathrm{L}}^{\text {in }}+\kappa \hat{\boldsymbol{p}}_{\mathrm{b}}^{\text {in }}+\kappa \sqrt{\varrho} \hat{\boldsymbol{w}}_{0}\right)+\sqrt{\epsilon} \hat{\boldsymbol{w}}_{1} \\
& \hat{\boldsymbol{p}}_{\mathrm{L}}^{\text {out }}=\sqrt{1-\epsilon} \hat{\boldsymbol{p}}_{\mathrm{L}}^{\text {in }}+\sqrt{\epsilon} \hat{\boldsymbol{w}}_{2} \\
& \hat{\boldsymbol{x}}_{\mathrm{b}}^{\text {out }}=\sqrt{1-\eta}\left(\hat{\boldsymbol{x}}_{\mathrm{b}}^{\text {in }}+\kappa \hat{\boldsymbol{p}}_{\mathrm{L}}^{\text {in }}\right)+\sqrt{\eta} \hat{\boldsymbol{w}}_{3} \\
& \hat{\boldsymbol{p}}_{\mathrm{b}}^{\text {out }}=\sqrt{1-\eta} \hat{\boldsymbol{p}}_{\mathrm{b}}^{\text {in }}+\sqrt{\eta} \hat{\boldsymbol{w}}_{4} .
\end{aligned}
$$

Here we identify $\hat{\boldsymbol{w}}_{n}(0 \leq n \leq 4)$ as standard vacuum-noise operators which correspond to normalized quantum-Weiner processes, satisfying $\left\langle\hat{\boldsymbol{w}}_{n}\right\rangle=0$ and $\left\langle\hat{w}_{m \alpha} \hat{w}_{n \beta}\right\rangle=\frac{1}{2} \delta_{m n} \delta_{\alpha \beta}$ for $\alpha, \beta \in\{y, z\}$ and $0 \leq m, n \leq 4$ [S11].

To estimate the attainable degree of squeezing and choose the optimal feedback pulse, we calculate the variance of the atomic spins after the feedback $\operatorname{var}\left(p_{A, i}^{\text {out }}+G x_{L, z}^{\text {out }}\right)$ and find that it attains a minimal value of

$$
\operatorname{var}\left(p_{A, i}^{\text {out }}+G x_{L, z}^{\text {out }}\right)=\frac{1}{2}\left(\frac{\kappa^{2}(1-\epsilon)(\eta+\varrho)+1}{\kappa^{2}(1-\epsilon)(1+\varrho)+1}\right)
$$

for the feedback proportionality constant

$$
G=-\frac{\kappa \sqrt{1-\epsilon} \sqrt{1-\eta}}{\left(1+\kappa^{2}(\varrho+1)(1-\epsilon)\right)} .
$$

\section{Appendix B: Entanglement lifetime}

In this section, we show that the variance of the squeezed quadrature of the two ensembles decays at the rate $2 \Gamma_{\mathrm{b}}$. In our case, the two independently squeezed quadratures are $\hat{p}_{\mathrm{b}, y}(t)$ and $\hat{p}_{\mathrm{b}, z}(t)$, which according to Eq. (S15) satisfy the dynamics

$$
\partial_{t} \hat{\boldsymbol{p}}_{\mathrm{b}}=-\Gamma_{\mathrm{b}} \hat{\boldsymbol{p}}_{\mathrm{b}}+\hat{\boldsymbol{F}}_{\mathrm{b}-}
$$

where $\left\langle\hat{\boldsymbol{F}}_{\mathrm{b}-}\right\rangle=0$, and

$$
\left\langle\hat{F}_{\mathrm{b}-, \alpha}\left(t-t^{\prime}\right) \hat{F}_{\mathrm{b}-, \beta}\left(t-t^{\prime \prime}\right)\right\rangle=\Gamma_{\mathrm{b}} \delta\left(t^{\prime \prime}-t^{\prime}\right)
$$

for $\alpha, \beta \in\{y, z\}$. Integration of Eq. S33 yields

$$
\hat{\boldsymbol{p}}_{\mathrm{b}}(t)=e^{-\Gamma_{\mathrm{b}} t} \hat{\boldsymbol{p}}_{\mathrm{b}}(0)+\int_{0}^{t} e^{-\Gamma_{\mathrm{b}}\left(t-t^{\prime}\right)} \hat{\boldsymbol{p}}_{\mathrm{b}}\left(t^{\prime}\right) d t^{\prime},
$$

where the second integral represents a standard stochastic integration. We first note that the initial vacuum-squeezed state is not displaced yielding $\left\langle\hat{p}_{\mathrm{b} \alpha}(0)\right\rangle=0$ and this $\left\langle\hat{p}_{\mathrm{b} \alpha}(t)\right\rangle=0$. To calculate the variance as a function of time we first find

$$
\hat{p}_{\mathrm{b} \alpha}^{2}(t)=e^{-2 \Gamma_{\mathrm{b}} t} \hat{p}_{\mathrm{b} \alpha}^{2}(0)+\int_{0}^{t} e^{-\Gamma_{\mathrm{b}}\left(2 t-t^{\prime}\right)}\left\{\hat{p}_{\mathrm{b} \alpha}(0), \hat{F}_{\mathrm{b}-, \alpha}\left(t^{\prime}\right)\right\} d t^{\prime}+\int_{0}^{t} \int_{0}^{t} e^{-\Gamma_{\mathrm{b}}\left(2 t-t^{\prime}-t^{\prime \prime}\right)} \hat{F}_{\mathrm{b}-, \alpha}\left(t^{\prime}\right) \hat{F}_{\mathrm{b}-, \alpha}\left(t^{\prime \prime}\right) d t^{\prime} d t^{\prime \prime}
$$




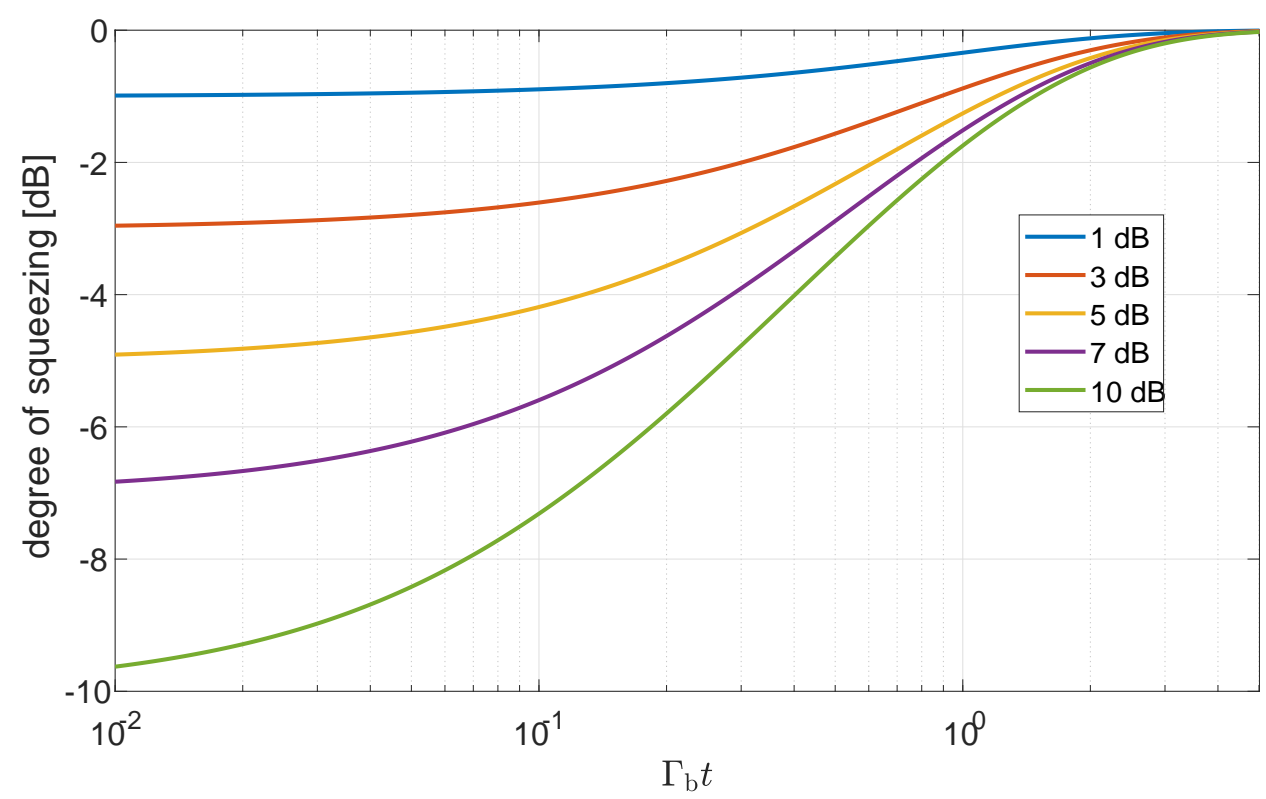

Figure S1. Degree of squeezing for vacuum-squeezed states with initial values of squeezing of $1,3,5,7$ and $10 \mathrm{~dB}$. While in a linear scale the variance decays at a constant rate $2 \Gamma_{\mathrm{b}}$, representation in a logarithmic $(\mathrm{dB})$ scale gives the time dependence shown

where $\{\cdot\}$ denotes the anti commutator. We can than calculate the variance by

$$
\begin{aligned}
\operatorname{var}\left(\hat{p}_{\mathrm{b} \alpha}(t)\right) & =e^{-2 \Gamma_{\mathrm{b}} t}\left\langle\hat{p}_{\mathrm{b} \alpha}^{2}(0)\right\rangle+\int_{0}^{t} \int_{0}^{t} e^{-\Gamma_{\mathrm{b}}\left(2 t-t^{\prime}-t^{\prime \prime}\right)}\left\langle\hat{F}_{\mathrm{b}-, \alpha}\left(t^{\prime}\right) \hat{F}_{\mathrm{b}-, \alpha}\left(t^{\prime \prime}\right)\right\rangle d t^{\prime} d t^{\prime \prime} \\
= & e^{-2 \Gamma_{\mathrm{b}} t}\left\langle\hat{p}_{\mathrm{b} \alpha}^{2}(0)\right\rangle+\Gamma_{\mathrm{b}} \int_{0}^{t} e^{-2 \Gamma_{\mathrm{b}}\left(t-t^{\prime}\right)} d t^{\prime} \\
= & \frac{1}{2}+\left(\left\langle\hat{p}_{\mathrm{b} \alpha}^{2}(0)\right\rangle-\frac{1}{2}\right) e^{-2 \Gamma_{\mathrm{b}} t}
\end{aligned}
$$

Therefore, the variance of a squeezed state initially with $\left\langle\hat{p}_{\mathrm{b} \alpha}^{2}(0)\right\rangle<\frac{1}{2}$ would decay at the rate $2 \Gamma_{\mathrm{b}}$ (i.e. twice the individual decoherence rate), whereas $\operatorname{std}\left(\hat{p}_{\mathrm{b} \alpha}\right)$ decays at a rate $\Gamma_{\mathrm{b}}$. Note that if the degree of squeezing is represented in $\mathrm{dB}$ scale via the definition $10 \log _{10}\left(2 \operatorname{var}\left(\hat{p}_{\mathrm{b} \alpha}(t)\right)\right)$, then the decay would seem faster for higher degree of squeezing as demonstrated in Fig. S1 for different initial squeezing degrees.

\section{Appendix C: Additional experimental configurations}

The entanglement generation scheme can be realized with various alkali and noble-gas mixtures within a large range of experimental parameters. Here we consider two additional configurations using two cylindrical cells of length $L=5$ cm and cross-section $A=2 \mathrm{~mm}^{2}$. First, we consider entanglement of two ${ }^{129}$ Xe ensembles each comprising gaseous mixture of 5 Torr ${ }^{129} \mathrm{Xe}, 50$ Torr $\mathrm{N}_{2}, 650$ Torr $\mathrm{Ne}$, and a droplet of ${ }^{87} \mathrm{Rb}$ at $175^{\circ} \mathrm{C}$. The $\mathrm{N}_{2}$ serves for quenching, and the Ne serves as a buffer gas that also acts to break XeRb molecules. Optical pumping of the Rb spins with $R_{\mathrm{op}}=1.6 \gamma_{\mathrm{a}}$ yields $P_{\mathrm{a}}=0.62$ [with $q\left(3 / 2, P_{\mathrm{a}}\right)=1.22$ ]. The ${ }^{129} \mathrm{Xe}$ is hyperpolarized via SEOP to $P_{\mathrm{b}}=0.46$, and its expected decoherence time $\gamma_{\mathrm{b}}^{-1} \approx 5 \mathrm{sec}$ is dominated by collisions with alkali atoms [S29, S35]. Magnetic field of order $B_{1} \approx 20 \mathrm{mG}$ is applied. A $250-\mathrm{mW}$ probe detuned $980 \mathrm{GHz}$ from the optical line is measured via homodyne detection for $T=200$ msec. These yield $\kappa=1.8, \epsilon=0.28, \eta=0.22, \varrho=0.17$, and could generate $3 \mathrm{~dB}$ of two-mode squeezing $(\xi=0.34)$. After the generation of entanglement, the optical pumping is turned off, the alkali depolarizes quickly, and the entanglement between the noble-gas ensembles can persist for several seconds.

Second, we consider entanglement of two ${ }^{3} \mathrm{He}$ ensembles using a gaseous mixture of 50 Torr ${ }^{3} \mathrm{He}, 100$ Torr $\mathrm{N}_{2}$, and a droplet of $\mathrm{K}$ at $250^{\circ} \mathrm{C}$. Here $R_{\mathrm{op}}=1.1 \gamma_{\mathrm{a}}$ yields $P_{\mathrm{a}}=0.52$ [with $q\left(3 / 2, P_{\mathrm{a}}\right)=1.28$ ] and $P_{\mathrm{b}}=0.48$, assuming 
$\gamma_{\mathrm{b}}^{-1}=50$ hour. The 400-mW probe is detuned $1 \mathrm{THz}$ from the optical line, and $B_{1} \approx 70 \mathrm{mG}$. Homodyne detection for $T=2$ minutes yields $\kappa=2.9, \epsilon=0.3, \eta=0.12$ and $\varrho=0.02$, generating almost $6 \mathrm{~dB}$ of two-mode squeezing $(\xi=0.68)$, which could live for hours.

\section{Appendix D: dependence of the interaction strength on the optical depth}

In the limit $\epsilon, \eta, \varrho \ll 1$, the attainable entanglement is governed by $\kappa$ which characterizes the polarization rotation induced by the (noble-gas) spin-noise and imprinted on the probe signal with respect to the photon shot-noise A similar parameter, $\tilde{\kappa}$, governs the attainable entanglement between alkali-spin ensembles (in the absence of noblegas), where it is known that $\tilde{\kappa}^{2}=2 \gamma_{\mathrm{a}} T d$ [S11]. There, one assumes that the alkali relaxation $\gamma_{\mathrm{a}}$ is dominated by scattering of probe photons, and one identifies the resonant optical-depth $d$ as the primary resource for entanglement. Similarly in our scheme, when the relaxation inherited from the alkali atoms $\left(J^{2} / \Delta^{2}\right) \gamma_{\mathrm{a}}$ dominates $\Gamma_{\mathrm{b}}$, the relation $\kappa^{2}=2 \Gamma_{\mathrm{b}} T d$ also holds as we now derive in the off-resonance regime:

$$
\begin{aligned}
& \kappa^{2}=\frac{J^{2} Q^{2} T^{2}}{\Delta^{2}+\gamma_{\mathrm{a}}^{2}}=T^{2} \frac{M_{\mathrm{a}} M_{\mathrm{L}} a^{2}}{T^{2}} \frac{J^{2}}{\Delta^{2}+\gamma_{\mathrm{a}}^{2}}=a^{2} M_{\mathrm{a}} M_{\mathrm{L}} \frac{J^{2}}{\Delta^{2}+\gamma_{\mathrm{a}}^{2}} \\
& =\left(\frac{\Gamma_{\mathrm{e}}^{2} \sigma^{2}}{A^{2} \delta_{\mathrm{e}}^{2}(2 I+1)^{2}}\right) N_{\mathrm{a}}(I+1 / 2) M_{\mathrm{L}} \frac{J^{2}}{\Delta^{2}+\gamma_{\mathrm{a}}^{2}}=T\left(\frac{M_{\mathrm{L}} \sigma}{T(2 I+1) A} \frac{\Gamma_{\mathrm{e}}^{2}}{\delta_{\mathrm{e}}^{2}}\right) \frac{n_{\mathrm{a}} \sigma L}{2} \frac{J^{2}}{\Delta^{2}+\gamma_{\mathrm{a}}^{2}} \\
& =2\left(\frac{\gamma_{\mathrm{absp}}}{\gamma_{\mathrm{a}}} \frac{J^{2} \gamma_{\mathrm{a}}}{\Delta^{2}+\gamma_{\mathrm{a}}^{2}}\right) T d
\end{aligned}
$$

where we use the on-resonance cross-section of a hot vapor with homogeneous broadening as $\sigma=2 r_{\mathrm{e}} c f / \Gamma_{\mathrm{e}}$. We also identify the number of alkali atoms $N_{a}=n_{a} A L$ and the on-resonance pumping rate $R_{a}=M_{\mathrm{L}} \sigma /(T(2 I+1) A)$, including the slowing-down factor of polarized ensembles. The off-resonance absorption rate is then $\gamma_{\mathrm{absp}}=R_{a} \Gamma_{\mathrm{e}}^{2} /\left(4 \delta_{\mathrm{e}}^{2}\right)$. The optical-depth of a single cell is $d=n_{\mathrm{a}} \sigma L$.

Assuming that the alkali relaxation is dominated by absorption of probe photons, one can set $\gamma_{\mathrm{a}} \approx \gamma_{\mathrm{absp}}$. Further assuming that the dominant relaxation of the noble-gas is due to its coupling to the alkali, $\Gamma_{\mathrm{b}} \approx J^{2} \gamma_{\mathrm{a}} /\left(\Delta^{2}+\gamma_{\mathrm{a}}^{2}\right)$, yields the relation

$$
\kappa^{2} \approx 2 \Gamma_{\mathrm{b}} T d
$$

Therefore, the optical-depth of the alkali ensembles remains the primary resource also for entangling noble-gas spins. 